\title{
La Musealización del Arte y la Historia: estrategias discursivas y de comunicación, inclusión, transformación digital y participación en museos
}

Ana Tirado-de la Chica - Universidad de Jaén

0000-0001-5761-7288

Recepción: 19.09.2021 | Aceptado: 16.10.2021

Correspondencia a través de ORCID: Ana Tirado-de la Chica

iD $0000-0001-5761-7288$

Citar: Tirado-de la Chica, A (2021). La Musealización del Arte y la Historia: estrategias discursivas y de comunicación, inclusión, transformación digital y participación en museos. REIDOCREA, 10(35), 1-18.

Financiación: Diputación Provincial de Jaén y el Instituto de Estudios Giennenses, a través del Plan 2020 de Subvenciones a universidades y centros de investigación para la realización de proyectos de investigación.

Estudio de Investigación del "Proyecto de Humanidades Digitales y Musealización. El viaje del ilustrado Antonio Ponz al Reino de Jaén e Intendencia de las Nuevas Poblaciones (siglo XVIII)" de la Universidad de Jaén.

Resumen: Este trabajo aborda una revisión del tema de la musealización del arte y la historia en exposiciones y museos. Se ha realizado una revisión bibliográfica sistematizada basada en publicaciones de revistas científicas internacionales indexadas en los tres primeros cuartiles del SJR, del periodo de 2015 a 2021. La muestra comprende 267 artículos. Se han abordado dos niveles de análisis: descriptivo, basado en variables independientes del año de publicación, nombre de las revistas, índice de indexación, editorial, lugar de publicación y autoría; e interpretativo, basado en variables dependientes de los temas y contenidos de la muestra. Se han utilizado dos técnicas de análisis cualitativo: de clasificación y reducción de datos por codificación, y análisis de contenidos, utilizando la herramienta informática de Atlas.ti 8. En los resultados se muestran las características descriptivas de los artículos analizados, según variables independientes, y los resultados de temas y contenidos principales (comunicación, inclusión, TIC y participación). La discusión de los resultados y conclusiones abordan una revisión crítica sobre las características de cada tema y aportan información sobre los retos futuros para la musealización del arte y la historia en exposiciones y museos.

Palabra clave: Musealización

Musealising Art \& History: narrative and communication strategies, inclusion, digital transformation and participation in museums

Abstract: This work addresses a review about musealising art and history in exhibitions and museums. It carries out a systematic bibliographic review based on international scientific journals that are indexed in the SJR's first three quartiles, from the period 2015 to 2021 . The sample comprises 267 articles. It approached two levels of analysis: one descriptive analysis, based on independent variables like the year of publication, name of the journals, indexing index, publisher, place of publication and authorship; and other interpretive analysis, based on dependent variables on the topics and contents of the sample. It used two qualitative analysis techniques: a data classification and reduction by coding and a content analysis, using the Atlas.ti8 computer tool. Findings show the descriptive characteristics of the articles analysed, according to independent variable, and findings about main topics and contents (communication, inclusion, ICT and participation). The discussion of the results and conclusions address a critical review of the characteristics of each topic and provide information on future challenges for musealising art and history in exhibitions and museums.

Keyword: Musealising

\section{Introducción}

Este trabajo nos preguntamos por las tendencias actuales de la musealización del arte y la historia en exposiciones y museos. En los últimos años estamos asistiendo a nuevos fenómenos en el acceso y exposición del patrimonio artístico y cultural, y es momento de una reflexión crítica del sector. En el año 2018, pudo verse en el Pabellón de la 
Navegación de Sevilla (España), la exposición de Van Gogh Alive: The Experience ${ }^{1}$, que consistió en un exposición de tipo inmersiva, donde muy variadas imágenes de la obra del artista se reproducían a gran escala, en pantallas de varios metros extensión. Los museos están abriendo canales en todas las redes sociales populares. Además de Facebook, Twitter, YouTube e Instagram, desde 2020 los museos también están presentes en TikTok, creando y publicando contenidos audiovisuales específicos del canal (Marín-Cepeda, 2021). Asimismo, la definición misma de museo se encuentra actualmente en debate en los comités de especialización del ICOM. En los resultados de la segunda consulta realizada (ICOM, 2021), se han identificado cuatro términos principales con frecuencias por encima del $70 \%$ de las respuestas, que son: investigación, conservación/preservación, patrimonio y educación/didáctica (p. 6). Otros términos, con frecuencias del $60 \%$, han sido: inclusivo, colección, y exponer/exhibir ( $\mathrm{p}$. $6)$.

La musealización es un término muy empleado en el sector, pero que con poca frecuencia se ha abordado en un sentido conceptual o epistemológico del término. láñez-Ortega, (2014) lo emplea sistemáticamente para referirse a la musealización del patrimonio cinematográfico. Distingue entre la simple consulta, acceso o reproducción en exposición de las obras cinematográficas, que son las acciones culturales propias de las filmotecas en su función de archivos, de un montaje museográfico sobre el fenómeno del cine en sus diferentes aspectos (óptico, social, tecnológico, etc.), como es el caso del Museo del Cine en Gerona ${ }^{2}$. Entendiendo entonces el museo como un lugar de musealización, "la exposición es la visualización explicativa de hechos ausentes por medio de los objetos, así como de elementos de la puesta en escena, utilizados como signos" (Schärer, 2003, citado en Desvallées y Mairesse, 2010, p. 37). Por tanto, la musealización comprende una amplia variedad de estrategias que convergen con la comunicación (Martín-Cáceres y Cuenca-López, 2016), la performatividad del espacio (Diamantopoulou y Christidou, 2016), la educación, etc. Se trata así de un ámbito complejo y en el que además han irrumpido las TIC y otras cuestiones sociales, como la inclusión, que desconstruyen poco a poco los paradigmas anteriores de la musealización, hacia nuevas formas de difundir y comunicar el patrimonio con la sociedad. En este sentido, el propósito principal de esta investigación es realizar un estado actual del tema de la musealización del arte y la historia, de acuerdo a publicaciones periódicas científicas indexadas en el ranking internacional de impacto del SCImago Journal Rank (SJR, en adelante). En primer lugar, en el apartado de Método, se realiza una descripción detallada del procedimiento de revisión bibliográfica sistematizada que se ha realizada; y se describe el procedimiento de análisis, según técnicas de codificación y de análisis de contenido de metodologías cualitativas. En segundo lugar, en el apartado de Resultados, se muestran los resultados del análisis descriptivo e interpretativo, de acuerdo a los dos grupos de variables establecidos, respectivamente. Finalmente, en tercer lugar, se realiza una discusión de los resultados sobre las características de cada tema y los retos para la musealización del arte y la historia en exposiciones y museos.

\section{Objetivos de estudio}

El objetivo principal de este trabajo es conocer el estado actual de los estudios de la musealización del arte y la historia en publicaciones periódicas científicas de alto impacto. Para ello, se establecen tres objetivos específicos de la investigación:

\footnotetext{
${ }^{1}$ https://www.pabellondelanavegacion.com/web/centro-expositivo/van-gogh-alive

${ }^{2}$ https://museudelcinema.girona.cat/esp/museu info.php
} 
1. Analizar el ámbito de las publicaciones de estudio de musealización de arte e historia en publicaciones periódicas científicas internacionales.

2. Analizar los temas y contenidos principales que se abordan en los estudios de musealización de arte e historia en publicaciones periódicas científicas internacionales.

3. Interpretar las tendencias principales en la investigación de la musealización de arte e historia en publicaciones periódicas científicas internacionales.

\section{Método}

De acuerdo al objetivo conocer el estado actual de los estudios de la musealización del arte y la historia, se realiza una revisión bibliográfica sistematizada en el ámbito de la museología, siguiendo los ejemplos anteriores de Ayala, Cuenca-Amigo y Cuenca (2019) y de Álvarez-Rodríguez, Marfil-Carmona y Báez-García (2019). Para el método, se ha utilizado el establecido por Bettany-Saltikov (2012), que comprende cuatro pasos: (1) Criterios de inclusión y exclusión, (2) Estrategia de búsqueda, (3) Selección publicaciones y (4) Extracción de datos (también contempla realizar una revisión de la calidad metodológica de las publicaciones, pero esta se ha integrado en los criterios de inclusión y exclusión, de acuerdo al cuartil de indexación de la publicación).

\section{Criterios de inclusión y exclusión:}

Se establece como fuente principal de información para una revisión del estado actual del tema de la musealización del arte y la historia en exposiciones y museos, los artículos publicados en revistas científicas internacionales de alto impacto. Para ello, se emplean dos bases de datos bibliográficos especializados principales: el SJR, sobre el ranking internacional de indexación de revistas SJR, y las plataformas en línea de la editorial Taylor \& Francis Online y de la Web of Science (WoS, en adelante), sobre la edición de revistas de alto impacto especializadas en temas de museos ${ }^{3}$.

Para la periodicidad de las publicaciones, y dado que se realiza una revisión actual del tema de estudio, se ha establecido una periodicidad de los últimos cinco años, según trabajos publicados entre 2015 y 2020 . También se han incluido las publicaciones del año en curso de 2021, según estuvieran disponibles en la fase de recogida de datos de esta investigación.

Para el criterio de alto impacto de las publicaciones, se establecen la clasificación de las revistas en los primeros cuartiles del SJR, según Q1, Q2 o Q3. Se incluye el tercer cuartil de la indexación (Q3), dado que la indexación en estos rankings de las publicaciones del ámbito de las Arte y Humanidades no se encuentra muy extendida, y existen revistas de gran reconocimiento y prestigio en el ámbito de los museos, pero que históricamente no se han clasificado en los principales cuartiles internacionales.

\section{Estrategia de búsqueda:}

\footnotetext{
${ }^{3}$ Se ha descartado utilizar el ranking Journal Citation Report (JCR), que constituye el índice principal del impacto internacional de las revistas científicas, porque este ranking, y a diferencia del SJR, no incluye una categoría específica de Museología en la clasificación temática de las revistas indexadas y, por tanto, el JCR ofrece el listado de revistas clasificas en categorías temáticas más generales, tales como "Art", "Cultural Studies" o "Humanities (multidisciplinary)". Así, los resultados de revistas clasificadas en estas categorías generales del JCR se cuentan por cientos y corresponden a disciplinas muy variadas, que, además, la mayoría de estas no son afines a temas de museos, A su vez, las revistas clasificadas en JCR también aparecen en los primeros cuartiles del SJR.
} 
En primer lugar, se realiza la búsqueda de revistas en el SJR. Para ello, se establece una primera selección directa de revistas según la categoría de Museología. Esta corresponde a 41 revistas con indexación de cuartiles Q1, Q2 y Q3. Sobre estas, se han aplicado algunos criterios de exclusión. Se han descartado las revistas con temática exclusiva en Arqueología, Historia Natural, Conservación, ya que no ofrecen resultados de artículos sobre bienes culturales de arte e historia, según son objeto de estudio en este trabajo. También se han descartado las revistas donde los textos completos de los artículos están publicados en idiomas que no son legibles por la investigadora, esto es, diferentes al inglés, el español, el portugués, el francés o el italiano. Así, el número de revistas seleccionadas para la búsqueda es de 17 revistas. Se añadió también una última revista que es muy popular en el ámbito de los museos, pero que no están indexadas en la categoría de "Museology", sino de Visual Arts and Performing Arts del SJR, que es: Museum, Management and Curatorship (Q1). De este modo, el número total de revistas seleccionadas es de 18 revistas, que constituyen las revistas de búsqueda de artículos publicados desde 2015 hasta la actualidad, con alta indexación en SJR que aborden temas específicos de la musealización de arte e historia.

En segundo lugar, se han realizado dos procedimientos de búsqueda de artículos sobre las bases de archivos bibliográficos de cada revista de la selección anterior. Por un lado, revisando directamente los índices en el histórico de volúmenes publicados, de acuerdo a la selección anterior de revistas. Así, se han revisado los índices de cada volumen publicados en el periodo de estudio, desde 2015 hasta la actualidad.

Por otro lado, para la búsqueda en la base de datos bibliográficas de WoS y Taylor \& Francis, se han utilizado palabras clave de búsqueda. Las palabras clave de búsqueda se han definido de acuerdo a los objetivos de este estudio, que han sido: museum, musealising, exhibition, display, curat, narrative, communication, communicate, art, history. En general, el idioma de las palabras que se ha utilizado es el inglés, dado que los títulos y resúmenes de artículos publicados en revistas internacionales se publican traducidos al inglés, incluso cuando el idioma principal de la publicación es otro.

\section{Selección de publicaciones:}

Para la selección de artículos para la muestra, se estableció como criterio general que el tema principal fuera relativo y afín a la musealización en la difusión de contenidos artísticos e históricos del patrimonio cultural. La selección se realizó de manera inductiva sobre el título y resumen de los artículos. Se descartaron los artículos de revisión de libros. Sí se incluyeron los artículos de revisión de exposiciones.

\section{Extracción de datos:}

Para la recogida de los artículos, se utilizó el gestor bibliográfico de RefWorks ${ }^{4}$. Este dispone de una opción para importar nuevas referencias directamente desde la URL o DOI de las publicaciones, de manera que permite añadir las referencias de los artículos en línea seleccionados desde los índices de las revistas digitales. Al terminar la recogida de artículos, se hizo una comprobación de duplicados, según la opción de comprobación directa que dispone RefWorks. También se realizó una comprobación de datos bibliográficos pues, en ocasiones, se producen errores de importación de datos y pueden quedar algunos campos en blanco. Esta comprobación no es automática en RefWorks. Para ello se empleó el programa informático de Microsoft Excel 2010. Primero se realizó una exportación bibliográfica de RefWorks en archivo Excel. A continuación, con la herramienta de Tabla dinámica de Microsoft Excel 10, se pudieron

\footnotetext{
${ }^{4}$ https://refworks.proquest.com/
} 
revisar los campos en blanco y recoger manualmente los datos bibliográficos que faltaran.

Análisis de datos:

Se realizaron dos niveles de análisis. Un primer nivel descriptivo sobre las características principales de los artículos recogidos, de acuerdo a una serie de variables independientes ( $\mathrm{VI}$, en adelante) establecidas de manera deductiva. $\mathrm{Y}$ un segundo nivel interpretativo sobre los contenidos temáticos principales de los artículos, de acuerdo a variables dependientes (VD, en adelante), establecidas de manera inductiva.

Para el análisis descriptivo, se realizó un análisis descriptivo basado en el cálculo de frecuencias de las VI, que se establecieron en siete, según las siguientes: autoría (nombre de autores), cronología (según los siguientes indicadores del año de publicación: 2015, 2016, 2017, 2018, 2019, 2020 o 2021, publicación (nombre de la revista), editorial (nombre de la editorial), lugar de publicación (nombre del país), título y resumen. Para este cálculo, se utilizó la herramienta de tabla dinámica de Microsoft Excel 2010, que se aplicó, a su vez, sobre el archivo de datos bibliográficos exportado de RefWorks, según se había realizado anteriormente en la recogida de artículos.

Para el análisis interpretativo, se utilizó un método cualitativo de análisis de contenido, basado en la técnica de reducción de datos por codificación (Miles, Huberman y Saldaña, 2019; Bazeley, 2013), que esencialmente consistió en clasificar los documentos de artículos recogidos por categorías temáticas y, a continuación, establecer contenidos principales respecto de cada una de ellas. Para ello, se utilizó la herramienta informática de Atlas.ti 8 , que soporta información en formato de texto (documentos escritos) e integra herramientas específicas para el análisis cualitativo. El procedimiento de codificación se realizó en varias fases sucesivas. En primer lugar, se realizó una primera clasificación de los artículos en temáticas generales, basada los términos más frecuentes de la muestra. Para ello, se realizó la herramienta de cálculo de frecuencia de palabras de Atlas.ti 8, que se aplicó sobre las VI de título y resumen de los artículos. Estas fueron: "art", "digital", "exhibition", "social", "visitors", "national", "history", "public", design", "experiences", entre otras (ver Figura 1).

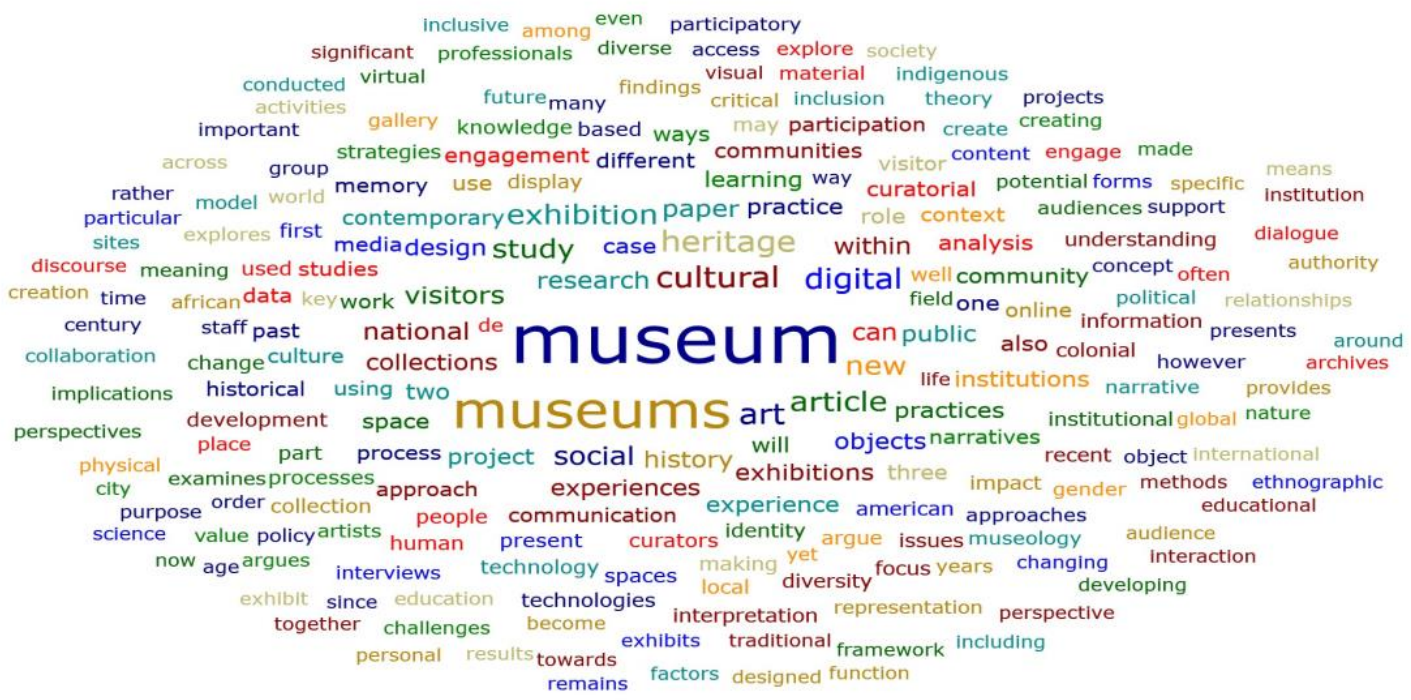

Figura 1. Resultados de la frecuencia de palabras en el conjunto de la muestra. 
A continuación, se realizó una segunda codificación, revisando la anterior, y estableciendo a su vez nuevas categorías, que aún fueron de tipo general. Por tanto, se continuó el procedimiento con una tercera codificación para contenidos específicos de los grupos temáticos anteriores. Para ello se utilizó la herramienta de grupos de documentos, agrupando los artículos de acuerdo a categorías temáticas generales. Para finalizar, se realizó una revisión conjunta, para evitar repeticiones de códigos, de citas, de documentos, y de documentos o citas sin codificar.

\section{Resultados}

En primer lugar, se muestran los resultados relativos al análisis descriptivo, de acuerdo a las variables independientes de cronología, revista, indexación de la publicación, editorial y lugar de publicación. En segundo lugar, se muestra los resultados del análisis interpretativo, de acuerdo a las variables dependientes relativas a los temas y contenidos de la muestra analizada. En total, se han analizado 267 artículos sobre el tema de musealización del arte y la historia de los últimos cinco años. A continuación, se muestra los resultados para sus características principales.

Según la cronología de las publicaciones, los resultados indican que son constantes y regulares a lo largo de los años de estudio, incluido lo que llevamos del actual de 2021. Se observa un aumento progresivo, con mayor énfasis en 2020. Así, mientras que en 2015 se publicaron 28 artículos para el tema de musealización del arte y la historia, en 2019 se publicaron 42 artículos y, en 2020, 72 artículos (ver Tabla 1). Por tanto, la musealización del arte y la historia es un tema de gran actualidad e interés en el sector cultural de los museos.

Tabla 1. Resultados de frecuencia del año de publicación de los artículos de la muestra.

\begin{tabular}{|c|c|c|}
\hline Año de Publicación & № artículos & $\%$ artículos \\
\hline 2015 & 28 & 10,5 \\
\hline 2016 & 25 & 9,4 \\
\hline 2017 & 34 & 12,7 \\
\hline 2018 & 39 & 14,6 \\
\hline 2019 & 42 & 15,7 \\
\hline 2020 & 72 & 27,0 \\
\hline 2021 & 27 & 10,1 \\
\hline Total de artículos & 267 & 100 \\
\hline
\end{tabular}

Según la revista, los resultados indican hasta 22 revistas diferentes en los que se han publicado artículos para el tema de la musealización del arte y la historia. Se observa que la gran mayoría de los artículos se concentran en tan solo 4 revistas, con el $70 \%$ de la muestra (ver Tabla 2). Estas son: Museum Management and Curatorship, Curator: The Museum Journal, Museum International y International Journal of Heritage Studies. Por otro lado, según el índice de indexación del SJR (Q1, Q2 o Q3), los resultados indican que la mitad de los artículos están publicados en revistas Q1 (el 49,6\% de la muestra, según un total de 133 artículos entre 9 revistas), y que la otra mitad se encuentran más equitativamente repartidos entre revistas Q2 y Q3 (el 25,7\% de los artículos están publicados entre 7 revistas Q2; y el 24,6\% de los artículos están publicados entre 6 revistas Q3). 
Tabla 2. Resultados de frecuencia de las revistas de publicación de los artículos de la muestra.

\begin{tabular}{|c|c|c|c|c|}
\hline Núm. & Revistas de publicación & $\begin{array}{l}\text { Índice } \\
\text { SJR }\end{array}$ & $\begin{array}{l}\text { № } \\
\text { artículos }\end{array}$ & $\begin{array}{l}\% \\
\text { artículos }\end{array}$ \\
\hline 1 & Museum Management and Curatorship & Q1 & 58 & 21,7 \\
\hline 2 & Curator: The Museum Journal & Q1 & 50 & 18,7 \\
\hline 3 & Museum International & Q3 & 45 & 16,9 \\
\hline 4 & International Journal of Heritage Studies & Q2 & 34 & 12,7 \\
\hline 5 & Museum Anthropology & Q2 & 19 & 7,1 \\
\hline 6 & The Public Historian & Q2 & 10 & 3,7 \\
\hline 7 & Visitor Studies & Q1 & 9 & 3,4 \\
\hline 8 & Museums \& Social Issues & Q3 & 7 & 2,6 \\
\hline 9 & Museum Worlds: Advances in Research & Q3 & 6 & 2,2 \\
\hline 10 & $\begin{array}{l}\text { IL CAPITALE CULTURALE. Studies on the Value of Cultural } \\
\text { Heritage }\end{array}$ & Q3 & 6 & 2,2 \\
\hline 11 & Journal of Museum Education & Q1 & 5 & 1,9 \\
\hline 12 & International Journal of Cultural Policy & Q1 & 4 & 1,5 \\
\hline 13 & Perspectivas em Ciência da Informação & Q2 & 2 & 0,7 \\
\hline 14 & International Journal of Heritage Studies & Q1 & 2 & 0,7 \\
\hline 15 & Library Hi Tech & Q1 & 2 & 0,7 \\
\hline 16 & TransInformação & Q2 & 2 & 0,7 \\
\hline 17 & Applied Computing and Informatics & Q1 & 1 & 0,4 \\
\hline 18 & Collection and Curation & Q1 & 1 & 0,4 \\
\hline 19 & International Journal of Sociology and Social Policy & Q2 & 1 & 0,4 \\
\hline 20 & Visual Resources & Q3 & 1 & 0,4 \\
\hline 21 & International Journal of Event and Festival Management & Q3 & 1 & 0,4 \\
\hline 22 & Journal of Systems and Information Technology & Q2 & 1 & 0,4 \\
\hline \multicolumn{3}{|c|}{ Total de artículos } & 267 & 100 \\
\hline
\end{tabular}

Según la editorial, los resultados indican hasta 12 entidades o compañías diferentes dedicadas a la edición de estas publicaciones. Entre estas, Routledge presente una clara diferencia frente al resto. A sus revistas le corresponden más de la mitad de los artículos publicados (el 61,4\% de la muestra, según 164 artículos), (ver Tabla 3). También destacan otras tres editoriales, pero que presentan grandes distancias dos y tres veces inferiores respecto de Routledge: John Wiley \& Sons (el 18,7\% de la muestra y 50 artículos), y Phaedra Livingstone (el 7,1\% de la muestra y 19 artículos). Por otro lado, la información recogida sobre las editoriales también ha aportado información sobre la localización geográfica de estas respecto del país. En este sentido, las editoriales que se han encontrado como mayoritarias, a su vez, sitúan las publicaciones en los contextos anglosajones del Reino Unido (R.U.) y de Estados Unidos (EE.UU.) principalmente, con una representación de prácticamente el $80 \%$ de la muestra (ver Tabla 4). Otros de los países son Italia, Brasil y Países Bajos, aunque tienen representaciones minoritarias para el total de la muestra.

Tabla 3. Resultados de frecuencia de la editorial de publicación de los artículos de la muestra.

\begin{tabular}{|c|l|c|c|}
\hline \multicolumn{1}{|c}{ Núm. Editorial de publicación } & No artículos & $\%$ artículos \\
\hline 1 & Routledge & 164 & 61,4 \\
\hline 2 & John Wiley \& Sons, Ltd & 50 & 18,7 \\
\hline 3 & Phaedra Livingstone & 19 & 7,1 \\
\hline 4 & University of California Press & 10 & 3,7 \\
\hline 5 & Berghahn & 7 & 2,6 \\
\hline 6 & Universitá degli Studi di Macerata & 6 & 2,2 \\
\hline 7 & Emerald Publishing Limited & 5 & 1,9 \\
\hline 8 & Escola de Ciencia da Informacao da UFMG & 2 & 0,7 \\
\hline 9 & Pontifícia Universidade Católica de Campinas & 2 & 0,7 \\
\hline 10 & The University of Chicago Press & 1 & 0,4 \\
\hline 11 & Elsevier & 1 & 0,4 \\
\hline 12 & Emerald Group Publishing Ltd. Total de artículos & 267 & 61,4 \\
\hline \multicolumn{2}{|r|}{} & & 100 \\
\hline
\end{tabular}


Tabla 4. Resultados de frecuencia del país de publicación de los artículos de la muestra.

\begin{tabular}{|c|l|c|c|}
\hline \multicolumn{1}{c}{ Núm. } & \multicolumn{1}{c}{ País de publicación } & \multicolumn{1}{c}{ № artículos } & $\%$ artículos \\
\hline 1 & R.U. & 170 & 63,7 \\
\hline 2 & EE.UU. & 79 & 29,6 \\
\hline 3 & Italia & 7 & 2,6 \\
\hline 4 & EE.UU. y R.U. & 6 & 2,2 \\
\hline 5 & Brasil & 4 & 1,5 \\
\hline 6 & Países Bajos & 1 & 0,4 \\
\hline \multicolumn{2}{|r|}{ Total artículos } & 267 & 100 \\
\hline
\end{tabular}

Según la autoría de los artículos, los resultados indican una gran amplitud y variedad de autores/as que estudian y abordan el tema de la musealización del arte y la historia en la actualidad. Se han identificado hasta 472 autores/as diferentes entre el total de la muestra. Estos resultados se ven reforzados cuando se analiza la frecuencia de los/as autores/as. Así, tan solo 22 de los/as autores tienen autoría de más de un artículo en la muestra. El resto de los 450 autores/as tienen autoría de un solo artículo en la muestra. Entre esos/as 22 autores/as, el número máximo de artículos por autor/a no supera los tres artículos (ver Tabla 5), lo que confirma la heterogeneidad y variedad de los/as autores/as para este tema de estudio ${ }^{5}$.

Tabla 5. Resultados de frecuencia de los/as autores/as de los artículos de la muestra.

\begin{tabular}{|c|l|c|c|l|c|}
\hline \multirow{2}{*}{ № autor/a } & \multicolumn{1}{c}{ Apellido, Nombre } & \multicolumn{1}{c}{ № artículos } & \multicolumn{1}{c}{ № autor/a } & \multicolumn{1}{l|}{ Apellido, Nombre } & № artículos \\
\hline 1 & Christidou, Dimitra & 3 & 12 & Hayde, Donnelley & 2 \\
\hline 2 & Garlandini, Alberto & 3 & 13 & Anderson, S. & 2 \\
\hline 3 & Robinson, Helena & 3 & 14 & Macdonald, Sharon & 2 \\
\hline 4 & Nielsen, Jane K. & 2 & 15 & Culp, Katherine McMillan & 2 \\
\hline 5 & Legget, Jane & 2 & 16 & Neves, Joselia & 2 \\
\hline 6 & Gifreu-Castells, Arnau & 2 & 17 & Ride, Peter & 2 \\
\hline 7 & Bennett, Roger & 2 & 18 & Beaujot, Ariel & 2 \\
\hline 8 & Mason, Marco & 2 & 19 & Tzortzi, Kali & 2 \\
\hline 9 & Chipangura, Njabulo & 2 & 20 & Eardley, Alison F. & 2 \\
\hline 10 & Arrigoni, Gabriella & 2 & 21 & Françozo, Mariana & 2 \\
\hline 11 & Chiwara, Davison & 2 & 22 & Kottasz, Rita & 2 \\
\hline
\end{tabular}

Finalmente, se muestran los resultados del análisis interpretativo sobre los temas y contenidos principales de la muestra de artículos. Los resultados indican cinco grupos temáticos principales. En orden de mayor a menor representación en la muestra, estos son: Comunicación, Inclusión, TIC (Nuevas Tecnologías de la Información y de la Comunicación), Participación y Otros. Los cinco temas tienen una representación significativa en la muestra. Sin embargo, destaca el tema de la Comunicación como principal (34,5\%, con 92 artículos). Le siguen los temas de Inclusión y TIC, en torno al $20 \%$ y 50 artículos. Y, finalmente, Participación (16,1\%, con 43 artículos) y Otros (10,1\%, con 27 artículos), (ver Tabla 6).

\footnotetext{
${ }^{5} \mathrm{~A}$ este respecto, sería de interés otro estudio futuro que pudiera profundizar en las características de los/as autores/as según diferentes variables, como el género, la nacionalidad, la adscripción institucional, etc. En esta ocasión quedaba fuera de los límites de la investigación.
} 
Tabla 6. Resultados de temas principales de artículos de la muestra.

\begin{tabular}{|c|c|c|c|}
\hline № Grupo temático & Grupos temáticos & № artículos & \% Representación \\
\hline 1 & Comunicación & 92 & 34,5 \\
\hline 2 & Inclusión & 54 & 20,2 \\
\hline 3 & TIC & 51 & 19,1 \\
\hline 4 & Participación & 43 & 16,1 \\
\hline 5 & Otros & 27 & 10,1 \\
\hline & Total de artículos & 267 & 100 \\
\hline
\end{tabular}

Respecto de contenidos más específicos, los resultados indican hasta 63 contenidos diferentes entre el total de la muestra (ver Tablas de la 7 a la 11 del Anexo). Entre estos, 21 contenidos corresponden al tema de la Comunicación, entre los que destacan la comunicación nacional y hegemónica, multi-sensorial, de canonización y descanonización de narrativas hegemónicas, de micro-narrativas y de la violencia en narrativas del pasado, entre otras; 9 contenidos al tema de Inclusión, entre los que destacan LGBTQIA, la Inclusión racial y el género, entre otros; 18 contenidos del tema de las TIC, entre los que destaca la transformación digital de los museos y ejemplos de recursos tecnológicos que se están introduciendo en los museos para la comunicación con los públicos; 12 contenidos del tema de Participación, entre los que destaca el cocomisariado de las exposiciones como forma de participación. Finalmente, se han identificado Otros 3 contenidos, relativos a la teoría y política de los museos, los ecomuseos y la pandemia por covid-19.

\section{Discusión}

La musealización del arte y la historia es un tema de gran actualidad e interés en el sector cultural de los museos. Los resultados anteriores indican un aumento significativo de las publicaciones para este tema en los últimos cinco años. Así, mientras que para el año 2015 se han recogido 28 artículos, para el último año de 2020, han sido 72 artículos (ver Tabla 1). Los canales de difusión de estas publicaciones están muy concentrados en un número muy reducido de revistas y de los contextos anglosajones. La amplia mayoría de las publicaciones de este tema corresponden a tan solo cuatro revistas, y que, por tanto, se constituyen como principales: Museum Management and Curatorship, Curator: The Museum Journal, Museum International y International Journal of Heritage Studies (ver Tabla 2). Estas cuatro tienen indexación de primer cuartil del SJR y se editan en los contextos anglosajones del Reino Unido y de Estados Unidos, principalmente, así como en lengua inglesa, (ver Tablas 3 y 4). Sin embargo, la variedad de los/as autores/as es muy amplia, según se han distinguido hasta 472 autores/as diferentes de la muestra, que, a su vez, tan solo se han repetido en 22 autores/as, y con frecuencias muy reducidas, de apenas 3 o 2 artículos del/a mismo/a autor/a (ver Tabla 5). En este sentido, para estudios futuros, sería de interés profundizar en la hipótesis de si los contextos anglosajones de la edición y difusión de las publicaciones condiciona determinados aspectos como los contextos y casos de estudio, los enfoques metodológicos, entre otros. En esta ocasión, no se ha recogido información específica al respecto, aunque sí se ha constatado, sin tener una información detallada, que los casos de estudio han sido internacionales de muy diferentes contextos en el mundo, y también las metodologías.

Entre los 4 temas principales que se han identificado (Comunicación, Inclusión, TIC y Participación), se observa que el más recurrido ha sido el de la Comunicación del museo (92 artículos y el $34,5 \%$ de la muestra) y, de este, el del enfoque político de la comunicación del museo (ver Tabla 6). Esto indica que el tema mayoritario de estudio e 
investigación siguen siendo los temas teórico tradicionales de los museos, según los usos discursivos y políticos de los museos; y, en cambio, los nuevos giros y cambios paradigmáticos de los museos, siguen sin aterrizar ampliamente en el sector y, además, en la investigación se le dedica menor atención.

\section{La comunicación en la musealización del arte y la historia:}

La comunicación es una cuestión inherente al museo. Sin embargo, Nielsen (2017) señala que no se llega a entender cómo funciona, lo que redunda, a su vez, en ciertas lagunas para su implementación y mejora. El enfoque común que impera en estos estudios es el de una lectura crítica respecto de ese uso político de las colecciones y exposiciones en los museos. Howell (2015) parece haber dado en la clave en este sentido, cuando se refiere a que, a pesar de los visitantes de su muestra de estudio manifiestan un desapego sistemático por la narrativa nacional, en el ámbito político de los museos el discurso nacional se vuelve aún más prioritario. Baeza (2020) analiza cómo, en la retórica de la política cultural del museo de la National Gallery en Londres, se fomenta la democratización cultural y la emancipación ideológica de la audiencia, pero, paradójicamente, también se refuerza el discurso autoritario del Museo. Cabría entonces preguntarse, si precisamente el riesgo de que disminuya la noción de nación y de Estado entre la población, no será lo que provoca la intervención nacionalista de los discursos institucionales públicos.

Wrightson (2017) afirma que las prácticas museológicas participan de los discursos políticos colonialistas del reconocimiento, los cuales siguen perpetuando las narrativas coloniales de poder (por ejemplo, según realiza el estudio en el contexto de Canadá). La musealización del tema de la violencia también se utiliza en este sentido, por ejemplo, abstrayendo los objetos de armas hacia un tratamiento exclusivamente estético en la exposición, eludiendo las capacidades de daño y sufrimiento que provocan (Pauls y Walby, 2020). De esta forma, se provoca en el público sensaciones de gratitud e innovación, pero no de culpa. Levin (2020) expande esta idea, pero con un enfoque diferente. Este consiste en una forma expositiva más expresiva de la violencia, que persigue la sensibilización y la empatía del público. Sin embargo, termina provocando más bien lo contrario, cierto rechazo y confrontación. Así, la exposición o museos más expresivos de la violencia, se perciben como recluidos en sí mismos y opresivos. Un ejemplo de ello que mencionan el/a autor/a es el uso de imágenes de cuerpos dañados y sangrientos, que dejan al público abatido e intranquilo.

Las voces alternativas desde las que se construyen los discursos museológicos constituyen un aspecto clave en esta des-colonización. En los resultados del análisis, se han identificado dos estrategias que se están practicando en los últimos años. Por un lado, la musealización de historias locales, según a estas no les interesa la delimitación nacional del discurso, sino expandirse hacia relaciones más amplias y globales con el resto del mundo (Higashi, 2019). Geoghegan, Mcllvenna y van der Vaart (2017) identifican tres estrategias para desarrollar narrativas locales a partir de colecciones nacionales: la colaboración inter-institucional, la implicación de historiadores locales y la recogida de información local en eventos comunitarios e historias orales. También de los nuevos materiales culturales, como los del patrimonio intangible (Djabarouti, 2020), de la vida cotidiana (Morgan y Macdonald, 2020). Y, en lo que respecto más específicamente al ámbito de la museología, de la tipología de las casas-museo, según defiende Mikula (2015). En este mismo sentido, Sunnucks et al., (2020) defienden que las museologías locales forman parte de la ruptura de los discursos hegemónicos, que los/las autores/as identifican con "el ala derecha del populismo, el racismo sistemático y la cultura neoliberal de las guerras" (p. 168). Y, por otro lado, aumentando y reforzando los vínculos y relaciones de las colecciones con las 
cuestiones sociales. Praët (2019) plantea que las narrativas demasiado formalistas, técnicas y concentradas en el aspecto material de las colecciones, tienen aplicaciones de poder en los museos. Así, se basa en casos de museos creados en contextos coloniales, en los que se ha explotado estas narrativas formales, mientras que en otros contextos a los que también se exportó el concepto de museo europeo, estos se crearon más mezclados con la diversidad cultural del lugar.

Sobre los procesos de memoria en el museo y las exposiciones, se encuentran varios aspectos que intervienen. Por un lado, las herramientas curatoriales para representar el pasado (Leonardi, 2021), los formatos de exposición (Sweetman, Hadfield y O'Connor, 2020) y los aspectos emociones y sensibles (Faba-Zuleta y Aedo-Gajardo, 2020). Sobre la exploración de la exposición en los museos, se identifican dos aproximaciones diferentes. Por un lado, una dirigida a disponer la exposición para múltiples niveles y tipos de interacción. $\mathrm{Y}$, por otro, otra esencialmente intuitiva y que provoca cierta deambulación estética y sensible de los visitantes. Respecto de la primera, en la muestra se ha recogido el caso de Madsen y Jensen (2021), cuyos resultados ofrecen cinco criterios clave para ese planteamiento expositiva, a saber: la temática simple, la mentalidad de usuario, la narrativa y el cierre narrativo. Respecto del segundo, se han recogido dos casos, el de Selwood y McEnaney (2020), que destacan las extraordinarias estrategias de marketing para esa manera de exposición, y el de Debary y Roustan (2017), que analizan el caso del Musée du Quai Branly en París, según constituye uno de los ejemplos más destacados de exposición permanente de museos concebidos para la exploración, y termina dando a entender que la ausencia de contextualización histórica y de información interpretativa termina provocando la ausencia o desvanecimiento de "los otros", esto es, de otras culturas, en favor de un discurso colonial francés.

Respecto del enfoque multi-sensorial de los museos y sus exposiciones, los resultados indican cuatro variantes temáticas, según la experiencia estética en el museo, el silencio, el tacto y aplicado al público en general, aunque no presenten necesidades por alguna discapacidad. Høffding, Rung y Roald (2020) defienden que las experiencias estéticas deben tenerse en cuenta en el mismo propósito de los museos de arte y las considera de acuerdo a las políticas de participación activa de los públicos. Mehzoud (2019) aborda estrategias performáticas y del discurso teatral aplicadas al diseño de narrativas en la exposición, entendiendo las narrativas en los museos como narrativas del paisaje y del lugar. Mason y Sayner (2019) plantean el silencio en el museo como parte integral del proceso de memoria. Christidou y Pierroux (2019) utilizó objetos de esculturas de arte para tocar y aporta resultados que contribuyeron especialmente a un mejor entendimiento de los procesos escultóricos. Wang y Lei (2016) proponen rompe con la dicotomía de la exposición según los dos tipos de exposición, ya sea "de los ojos" o "de las manos", y propone entonces un nuevo tipo de exposición según "de las mentes". En este sentido, pone el foco sobre las libertades de interpretación y de entendimiento de las obras a través de herramientas multi-sensoriales. Finalmente, Eardley, Dobbin, Neves y Ride, (2018) estudian cómo herramientas multi-sensoriales aplicadas en visitas en familia contribuyen a la experiencia y satisfacción en el museo.

\section{La inclusión en la musealización del arte y la historia:}

Isselhardt y Cross (2020) focalizan el problema de las lagunas en materia de inclusión y diversidad de los museos en la propia ignorancia del tema que hay en los museos, y de que los museos tienen que enfrentan su propia ignorancia al respecto. En este sentido, Prescha (2021) se refiere a que los museos abordan la inclusión como una tendencia, y no como un problema social en el que también están implicados. De este modo, los museos siguen incurriendo en lagunas al reconocimiento de la diversidad y 
de su distribución (Kinsley, 2016). La inclusión se refiere a una justifica social de muy diversas minorías, según discriminación por motivos étnicos y raciales, de discapacidad física y/o intelectual, de diversidad sexual, y otras minorías en riesgo de exclusión social.

Así, por ejemplo, uno de los temas de inclusión más recurridos ha sido el de el reconocimiento de la diversidad sexual y de género. En el año 2014 se creó la Guía LGBTQ de la American Alliance of Museums (AAM), (Leitch et al., 2016). El tema de LGBTQIA (Lesbian, Gay, Bisexual, Transgender, Queer, Intersex y Asexua/ ${ }^{\circ}$ ) se aborda desde muy diversos enfoques: desde la prevalencia del tema LGBTQIA en la actividad curatorial de los museos (Brow y Buckne, 2020); sobre dificultades para exponer estos temas en determinadas zonas del mundo y soluciones como las colaboraciones internacionales para exponer en itinerancia (Nhung, 2020); la educación de LGBTQIA en museos (Rowles, 2020; Kletchka, 2021), así como el patrimonio LGBTQI+(Bosold, Scott y Chantraine, 2020) y la historia de museos para este tema: sobre los ecosistemas de museos de Buenos Aires (Croizet, 2020), sobre el Museo Q en Colombia (ParraForero, 2020), y sobre el Museum of Sexual Diversity in São Paulo (Reinaudo, 2020).

Respecto del análisis e interpretación de las colecciones desde la perspectiva de género, Cuesta y Liliane (2020) distinguen dos tipos de estrategias, según: cuantitativa, sobre la prevalencia de los temas de mujeres, de obras de mujeres, y la ley de paridad, el estudio internacional comparado para la universalización de este problema (Assanova y Zhanguttin, 2020), entre otros; y cualitativa, permitiendo el cuestionamiento de los discursos y práctica del museo. En este último sentido, visibilizar el impacto de las mujeres en las narrativas de la historia es un tema destacado: en relación a objetos que fueron producidos por mujeres (Daybell, Heyam, Norrhem y Severinsson, 2020), la sobrerrepresentación del rol de mujeres en determinados ámbitos, como el trabajo de Heitman (2017) sobre las mujeres arqueólogas en la 1르 mitad del siglo XX.

\section{Las TIC en la musealización del arte y la historia:}

La transformación digital de los museos no es solo en apariencia. Está removiendo estas instituciones de la conservación y difusión del patrimonio desde sus raíces conceptuales. La era digital lleva implícita dos aspectos clave, entre otros: el acceso libre y abierto de los contenidos y también de la comunicación. En este sentido, las TIC favorecen una mayor democratización de la información y, también, una mayor extensión del rol de participación activa en el conjunto de la sociedad. Rivero (2019) reflexiona que, en este contexto, el rol de autoridad, de acumular los activos culturales por una minoría, que tradicionalmente han tenido los museos y sus agentes culturales (comisarios, artistas, críticos de arte, etc.) se desvanece. Y continúa afirmando que, el nuevo contexto de los museos en la era digital, es el cambio. La atención ya no está puesta en el aspecto material de las obras y las colecciones, sino en las capacidades narrativas que provocan. Las nuevas plataformas digitales de los museos (laboratorios en línea, bases de datos, sitios web), permiten el acceso abierto a la información artística $y$, de este modo, queda a expensas de una mayor crítica desde muy diferentes lugares del pensamiento y la cultura. Constituyen espacios de información, pero también y al mismo tiempo, espacios de comunicación colectiva y, en ocasiones, hasta en tiempo real. Por tanto, las TIC funcionan como un catalizador para el desarrollo y la innovación del sector cultural de los museos (Myrczik, 2020; Barranha, 2018). Sin embargo, la dicotomía entre museo físico y virtual sigue debatiéndose. Para Biedermann (2017), la

\footnotetext{
${ }^{6}$ Se escribe en inglés para evitar incurrir en la dicotomía de género del lenguaje escrito en español respecto de la sigla de LGBTQIA, que se refiere precisamente al reconocimiento de la diversidad sexual y de género.
} 
exposición virtual de la colección no sustituye a la tradicionales, y la plantea más bien como una herramienta independiente de aplicación educativa.

Entre los recursos TIC que se están introduciendo en los museos, en los resultados se han identificados unas dos docenas. Sin tener ocasión de poder abordar cada uno con mayor detalle, a continuación, se mencionan: aplicaciones para dispositivos móviles (Smartphone y Tablet), exposiciones virtuales, visitas remotas, tecnologías de 3D, la realidad aumentada, de realidad virtual, las redes sociales de museos, instalaciones digitales de luz, TIC inmersivas, códigos QR y robots.

\section{La participación en la musealización del arte y la historia:}

La participación en los museos se utiliza asociada a ejemplos muy heterogéneas de interacción. Robinson (2020) defiende que esto pone en evidencia que no existe consenso para unos principios fundamentales de la museología participativa y sobre uno criterios para su aplicación. Así, por ejemplo, y en el otro sentido, Coghlan (2018) plantea un concepto de la participación del museo, focalizada en la interacción y en las aportaciones del público sobre los significados, sentidos y valores de las cosas. Así, la participación sucede cuando los visitantes tienen la oportunidad de contribuir a la exposición, dejando un comentario, co-creando, etc. Poco a poco se están aportando contribuciones que van esclareciendo esta cuestión. Nowacki y Kruczek (2021) confirman que existe una relación entre la generación de contenidos, tener experiencias y la satisfacción de los visitantes con el Patrimonio.

En el comisariado de las exposiciones de museos se está produciendo un giro hacia una musealización más centrada en la atracción y participación de la ciudadanía. La autoridad y el liderazgo que tradicionalmente ha gozado el comisariado, actualmente está en cuestión, en favor de nuevas formas de musealizar y exponer más inclusivas. Unruh (2015) se refiere a un concepto de "comisariado dialógico" ("dialogical curating"), según se refiere a comisariar de manera colectiva y plural junto con el resto de agentes sociales y culturales en torno al tema u artefactos de exposición (Edmundson, 2017). En este mismo sentido, se han podido identificar varias estrategias concretas: utilizar acciones performáticas en el museo con comunidades sociales (Barnes y McPherson, 2019), investigar con técnicas etnográficas en las sociedades tradicionales y aborígenes los usos y significados de las cosas (Chipangura, 2020), utilizar una plataforma de diseño online para una co-creación de exposición con comunidades (Rogers y Rock, 2017), entre otras. Por otro lado, reconociendo los beneficios de este nuevo comisariado, Viau-Courville (2016) advierte de que también está desplazando la profesionalización del comisariado de las exposiciones.

En las conclusiones se identifican tres retos principales que enfrentan los museos en la musealización de su patrimonio artístico, cultural e histórico. En primer lugar, la descolonización de los discursos hegemónicos del poder, ya sea en clave nacional o de otro ámbito. La globalización de la economía, las ideas y las culturales, cada vez es más permeable a más capas o grupos de las sociedades, y esta no corresponde con narrativas centrales y de una sola voz. El segundo reto es la inclusión en sus diferentes vertientes (étnica, física, intelectual, cultural, social, etc.). La concepción de la inclusión en clave de justifica social aporta el enfoque desde que el que deben abordarse las alternativas a una musealización tradicional. En este sentido, el reto reside en hacer partícipes a otra variedad de voces, ideas y experiencias. Como alternativa, la musealización del arte y la historia debe introducir micro-narrativas y voces locales, con las que enriquecer sus interpretaciones y diversificar sus discursos. 
El tercer reto se refiere a un ámbito más museográfico o material de la musealización del arte y la historia, esto es, el de la transformación tecnológica de los recursos y dispositivos que se emplean para la musealización. En este sentido, el reto se refiere a la transformación digital y tecnológica de las exposiciones, incorporando las TIC y sus aportaciones. Sin embargo, el aparato o la tecnología en sí mismos no suponen una alternativa a los discursos hegemónicos y tradicionales del poder, por lo que las TIC deben ir de la mano, a su vez, de las estrategias de participación ciudadana. En este sentido, se buscan métodos de musealización más multi-sensoriales e interactivos.

\section{Referencias}

Álvarez-Rodríguez, D., Marfil-Carmona, R., y Báez-García, C. (2019). Investigación de impacto sobre la formación en mediación y educación en museos: análisis de la Web of Science. Revista Electrónica Interuniversitaria de Formación del Profesorado, 22(1), 121-144. https://doi.org/10.6018/reifop.22.1.357731

Assanova, D., y Zhanguttin, B. (2020). Women's museums in kazakhstan: Prospects in the new digital age. Museum International, 72(1-2), http://dx.doi.org/10.1080/13500775.2020.1743063

Ayala-Aizpuru, I., Cuenca-Amigo, M., y Cuenca-Amigo, J. (2019). Principales retos de los museos de arte en España. Consideraciones desde la museología crítica y el desarrollo de audiencias. Aposta. Revista de Ciencias Sociales, 80, 61-81. http://apostadigital.com/revistav3/hemeroteca/iayala.pdf

Baeza Ruiz, A. (2020). The rhetoric of looking: A case study about the exhibition of cleaned pictures of 1947. International Journal of Heritage Studies, 26(3), 237-252. https://doi.org/10.1080/13527258.2019.1620828

Barnes, P., y McPherson, G. (2019). Co-creating, co-producing and connecting: Museum practice today. Curator: The Museum Journal, 62(2), 257-267. https://doi.org/10.1111/cura.12309

Barranha, H. (2018). Derivative narratives: The multiple lives of a masterpiece on the internet. Museum International, 70(1-2), 22-33. https://doi.org/10.1111/muse.12190

Bazeley, P. (2013). Qualitative data analysis: practical strategies. SAGE.

Bettany-Saltikov, J. (2012). How to do a Systematic Literature Review in Nursing: a step-by-step guide. McGraw-Hill Education.

Biedermann, B. (2017). 'Virtual museums' as digital collection complexes. A museological perspective using the example of hansgross-kriminalmuseum. Museum Management and Curatorship, 32(3) 281-297. https://doi.org/10.1080/09647775.2017.1322916

Bosold, B., Scott, E., y Chantraine, R. (2020). Queer tactics, handwritten stories: Disrupting the field of museum practices. Museum International, 72(3-4), 212-225. https://doi.org/10.1080/13500775.2020.1873525

Brow, K., y Buckner, J. (2020). The "Rich gay"? small museums \& funding "Difficult" history. Curator: The Museum Journal, 63(4), 605610. https://doi.org/10.1111/cura.12388
Chipangura, N. (2020). Co-curation and new museology in reorganizing the beit gallery at the mutare museum, eastern zimbabwe. Curator: The Museum Journal, 63(3), 431-446. https://doi.org/10.1111/cura.12375

Christidou, D., y Pierroux, P. (2019). Art, touch and meaning making: An analysis of multisensory interpretation in the museum. Museum Management and Curatorship, 34(1), 96-115. https://doi.org/10.1080/096477775.2018.1516561

Coghlan, R. (2018). 'My voice counts because i'm handsome.' democratising the museum: The power of museum participation. International Journal of Heritage Studies, 24(7), 795809. https://doi.org/10.1080/13527258.2017.1320772

Croizet, F. (2020). LGBTQI+ representation in the museum ecosystem of Buenos Aires. Museum International, 72(3-4), 94-103. https://doi.org/10.1080/13500775.2020.1873499

Cuesta Davignon, L. I. (2020). Gender perspective and museums: Gender as a tool in the interpretation of collections. Museum International, 72(1-2), 80-91. https://doi.org/10.1080/13500775.2020.1743059

Daybell, J., Heyam, K., Norrhem, S., y Severinsson, E. (2020). Gendering objects at the V\&A and vasa museums. Museum International, 72(1-2), 106-117. https://doi.org/10.1080/13500775.2020.1779465

Debary, O., y Roustan, M. (2017). A journey to the musée du quai branly: The anthropology of a visit. Museum Anthropology, 40(1), 417. https://doi.org/10.1111/muan.12127

Desvallées, A. y Mairesse, F. (2010). Conceptos claves de museología. Armand Colin e ICOM. https://icom.museum/wpcontent/uploads/2018/07/Museologie_Espagnol_BD.pdf

Diamantopoulou, S., y Christidou, D. (2019). Museum encounters: A choreography of visitors' bodies in interaction. Museum Management and Curatorship, 34(4), 344-361. https://doi.org/10.1080/096477775.2019.1585279

Djabarouti, J. (2020). Stories of feelings and things: Intangible heritage from within the built heritage paradigm in the UK. International Journal of Heritage Studies, 27(4), 1-16. https://doi.org/10.1080/13527258.2020.1798271

Eardley, A. F., Dobbin, C., Neves, J., y Ride, P. (2018). Hands-on, shoes-off: Multisensory tools enhance family engagement within an art museum. Visitor $\quad$ Studies, 21(1), https://doi.org/10.1080/10645578.2018.1503873 
Edmundson, A. (2017). Beyond bricks and mortar: Reframing museum encounters. International Journal of Heritage Studies, 23(9),

https://doi.org/.1080/13527258.2017.1347578

Faba-Zuleta, P., y Aedo-Gajardo, Á. (2020). The task of the museum in shaping the aesthetic-political field of memory in postpinochet chile. Museum Anthropology, 43(2), 94-110. https://doi.org/10.1111/muan.12226

Geoghegan, H., Mcllvenna, K., y van der Vaart, M. (2017). Developing local narratives for objects in national collections: Lessons learned from the "Number please? working with the enfield exchange" project. Curator: The Museum Journal, 60(2), 217-233. https://doi.org/10.1111/cura.12201

Heitman, C. C. (2017). The Creation of Gender Bias in Museum Collections: Recontextualizing archaeological and archival collections from chaco canyon, new mexico. Museum Anthropology, 40(2), 128-142. https://doi.org/10.1111/muan.12139

Higashi, J. (2019). The politics of history in memorial museums. Museum International, 71(1-2), 96-103. https://doi.org/10.1080/13500775.2019.1638034

Høffding, S., Rung, M., y Roald, T. (2020). Participation and receptivity in the art museum: $A$ phenomenological exposition. Curator: The Museum Journal, 63(1), 69-81. https://doi.org/10.1111/cura.12344

Howell, D. R. (2015). Telling the story of welsh Art-But is anyone listening? Curator: The Museum Journal, 58(4), 423-433. https://doi.org/10.1111/cura.12138

láñez-Ortega, M. (2014). Musealización del patrimonio cinematográfico: los museos de cine en M. L. Bellido Gant, Arte y museos del siglo XXI: entre los nuevos ámbitos y las inserciones tecnológicas (pp. 37-52). Editorial UOC.

ICOM. (2021). El Museo: Informe sobre las opiniones de los miembros del ICOM para una nueva definición de museo. https://icom.museum/es/recursos/normas-y-directrices/definiciondel-museol

Isselhardt, T., y Cross, L. (2020). You love them, but you don't know them: Recognizing and welcoming lived experiences. Curator: The Museum Journal, 63(4), 571-578. https://doi.org/10.1111/cura.12390

Kinsley, R. P. (2016). Inclusion in museums: A matter of social justice. Museum Management and Curatorship, 31(5), 474-490. https://doi.org/10.1080/09647775.2016.1211960

Kletchka, D. C. (2021). The epistemology of the basement: A queer theoretical reading of the institutional positionality of art museum educators. Museum Management and Curatorship, 36(2), 125-135. https://doi.org/10.1080/09647775.2021.1894595

Leitch, C., Youngs, R., Gavigan, A., Lesperance, M., Burns, W. J., Cohen-Stratyner, B., y Hansen, J. (2016). LGBTQ welcoming guidelines for museums: Developing a resource for the museum field. Museums \& Social Issues, 11(2), 138-146. https://doi.org/10.1080/15596893.2016.1217460

Leonardi, F. (2021). Curating the context: Re-enacting and reconstructing exhibitions as ways of studying the past. Museum Management and Curatorship, 36(2), 172-188. https://doi.org/10.1080/09647775.2020.1803118
Levin, A. K. (2020). Crossing over: Museums as spaces of violence. Museum International, 72(3-4), 28-41. https://doi.org/10.1080/13500775.2020.1873492

Madsen, K. M., y Jensen, J. F. (2021). Learning through exploration at museum exhibitions. Museum Management and Curatorship, 36(2) 154-171. https://doi.org/10.1080/09647775.2020.1803115

Marín-Cepeda, S. (2021). El Museo en TikTok. En M. PallarésPiquer, J. Gil-Quintana y A. Santisteban-Espejo, Docencia, Ciencia y Humanidades: hacia una enseñanza integral en la universidad del siglo XXI (pp. 102-112). Dykinson, S.L.

Martín-Cáceres, M. J., y Cuenca-López, J. M. (2016). Communicating heritage in museums: Outlook, strategies and challenges through a SWOT analysis. Museum Management and Curatorship, 31(3), 299-316. https://doi.org/10.1080/09647775.2016.1173576

Mason, R., y Sayner, J. (2019). Bringing museal silence into focus: Eight ways of thinking about silence in museums. International Journal of Heritage Studies, 25(1), 5-20. https://doi.org/10.1080/13527258.2017.1413678

Mehzoud, S. (2019). Scenographic exhibitions as spaces of encounter. Curator: The Museum Journal, 62(4), 629-648. https://doi.org/10.1111/cura.12340

Miles, M. B., Huberman, M. A, y Saldaña, J. (2014). Drawing and Verying Conclusions. Qualitative Data Analysis: A Methods Sourcebook. SAGE.

Mikula, M. (2015). Vernacular museum: Communal bonding and ritual memory transfer among displaced communities. International Journal of Heritage Studies, 21(8), 757-772. https://doi.org/10.1080/13527258.2015.1020961

Morgan, J., y Macdonald, S. (2020). De-growing museum collections for new heritage futures. International Journal of Heritage Studies, 26(1), 56-70. https://doi.org/10.1080/13527258.2018.1530289

Myrczik, E. P. (2020). Cultivating digital mediation: The implementation of publicly funded digital museum initiatives in denmark. International Journal of Cultural Policy, 26(2), 239-254. https://doi.org/10.1080/10286632.2018.1495714

Nhung, D. T. (2020). Reflections on the making of the unstraight exhibitions. Museum International, 72(3-4), 42-53. https://doi.org/10.1080/13500775.2020.1873495

Nielsen, J. K. (2017). Museum communication and storytelling: Articulating understandings within the museum structure. Museum

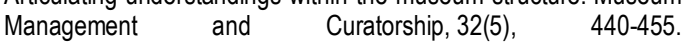
https://doi.org/10.1080/09647775.2017.1284019

Nowacki, M., y Kruczek, Z. (2021). Experience marketing at polish museums and visitor attractions: The co-creation of visitor experiences, emotions and satisfaction. Museum Management and Curatorship, 36(1), 62-81. https://doi.org/10.1080/09647775.2020.1730228

Parra-Forero, M. A. (2020). Museo Q: Museology in motion. Museum International, 72(3-4), 142-153. https://doi.org/10.1080/13500775.2021.1873503

Pauls, H., y Walby, K. (2020). Military, meaning, and tactical myopia: Representations of weapons at the royal canadian artillery 
museum. International Journal of Heritage Studies, 26(11), 11091122. https://doi.org/10.1080/13527258.2020.1752774

Praët, M. V. (2019). The modernity of the museum: Sharing and creating knowledge through confrontation with the material evidences of the past and the present. Museum International, 71(12), 38-47. https://doi.org/10.1080/13500775.2019.1638025

Prescha, L. (2021). Myth of neutrality and non-performativity of antiracism. Museum Management and Curatorship, 36(2), 109-124. https://doi.org/10.1080/09647775.2021.1891559

Reinaudo, F. (2020). A strange queer body: The museum of sexual diversity in são paulo, brazil. Museum International, 72(3-4), 16-27. https://doi.org/10.1080/13500775.2020.1873490

Rivero Moreno, L. D. (2019). Museums and digital era: Preserving art through databases. Collection and Curation, 38(4), 89-93. https://doi.org/10.1108/CC-02-2018-0002

Robinson, H. (2020). Curating good participants? Audiences, democracy and authority in the contemporary museum. Museum Management and Curatorship, 35(5), 470-487. https://doi.org/10.1080/09647775.2020.1803117

Rogers, A., y Rock, J. (2017). Testing a mobile platform for community co-created exhibitions. Curator: The Museum Journal, 60(3), 335-349. https://doi.org/10.1111/cura.12194

Rowles, B. (2020). LGBTIQ+-themed education at the kunsthistorisches museum in vienna - guided tours with a drag queen. Journal of Museum Education, 45(4), 364-374. https://doi.org/10.1080/10598650.2020.1814985

Selwood, S., y McEnaney, L. (2020). Exhibition reviews. Hogarth: Place and progress, sir john soane's museum, London, 9 october $2019-5$ january 2020. Museum Worlds: Advances in Research, 8 , 216-228. https://doi.org/10.3167/armw.2020.080116

Sunnucks, L. O., Levell, N., Shelton, A., Suzuki, M., Isaac, G., y Marsh, D. E. (2020). Interruptions: Challenges and innovations in exhibition-making. Museum Worlds: Advances in Research, 8, 168187. https://doi.org/10.3167/armw.2020.080112

Sweetman, R., Hadfield, A., y O'Connor, A. (2020). Material culture, museums, and memory: Experiments in visitor recall and memory. Visitor Studies, 23(1) 18-45. https://doi.org/10.1080/10645578.2020.1731671

Unruh, L. (2015). Dialogical curating: Towards aboriginal selfrepresentation in museums. Curator: The Museum Journal, 58(1), 77-89. https://doi.org/10.1111/cura.12099

Viau-Courville, M. (2016). Museums without (scholar-)curators: Exhibition-Making in times of managerial curatorship. Museum International, 68(3-4), 11-32. doi:10.1111/muse.12126

Wang, Q., y Lei, Y. (2016). Minds on for the wise: Rethinking the contemporary interactive exhibition. Museum Management and Curatorship, 31(4)

331-348.

https://doi.org/10.1080/09647775.2016.1173575

Wrightson, K. R. (2017). The limits of recognition: The spirit sings, canadian museums and the colonial politics of recognition. Museum Anthropology, 40(1), 36-51. https://doi.org/10.1111/muan.12129 
Tabla 7. Resultados de temas y contenidos para el grupo temático de Comunicación.

\begin{tabular}{|c|l|c|c|}
\hline $\begin{array}{c}\text { No temas y } \\
\text { contenidos }\end{array}$ & Temas y contenidos & \multicolumn{3}{c|}{ № } & artículos & Grupos temáticos \\
\hline 1 & Nacional, hegemónica & 18 & Comunicación \\
\hline 2 & Multi-sensorial & 14 & Comunicación \\
\hline 3 & Canonización y descanonización de narrativas hegemónicas & 9 & Comunicación \\
\hline 4 & Intervención de artista(s) & 5 & Comunicación \\
\hline 5 & Micro-narrativas & 5 & Comunicación \\
\hline 6 & Violencia en narrativas del pasado & 5 & Comunicación \\
\hline 7 & La construcción de la memoria en el museo y la exposición & 4 & Comunicación \\
\hline 8 & La narrativa del espacio y escenografía en la exposición & 4 & Comunicación \\
\hline 9 & Criterios que condicionan las decisiones del comisariado & 4 & Comunicación \\
\hline 10 & Ordenar y disponer obras en exposición & 4 & Comunicación \\
\hline 11 & Características de la experiencia de exploración de la exposición & 3 & Comunicación \\
\hline 12 & Condiciones profesionales de la narrativa e interpretación & 3 & Comunicación \\
\hline 13 & Estrategias de aprendizaje aplicadas al diseño de la exposición & 2 & Comunicación \\
\hline 14 & Experiencia estética & 2 & Comunicación \\
\hline 15 & Nuevos espacios de trabajo del museo abiertos al visitante & 2 & Comunicación \\
\hline 16 & Performatividad del espacio & 2 & Comunicación \\
\hline 17 & Teoría del museo desde el enfoque de la comunicación & 2 & Comunicación \\
\hline 18 & Diseñar la visualización de datos para exposiciones & 1 & Comunicación \\
\hline 19 & La necesidad de agregar la historia de los objetos en la exposición & 1 & Comunicación \\
\hline 20 & Narrativa transmedia & 1 & Comunicación \\
\hline 21 & Los textos de los museos para infantil & 1 & Comunicación \\
\hline & & 92 & Comunicación \\
\hline
\end{tabular}

Tabla 8. Resultados de temas y contenidos para el grupo temático de Inclusión.

\begin{tabular}{|c|l|c|c|}
\hline \multirow{2}{*}{$\begin{array}{l}\text { № temas y } \\
\text { contenidos }\end{array}$} & Nemas y contenidos & 12 & Grupo temático \\
\hline 1 & LGBTQIA & 10 & Inclusión \\
\hline 2 & Inclusión racial & 9 & Inclusión \\
\hline 3 & Género & 8 & Inclusión \\
\hline 4 & Accesibilidad & 8 & Inclusión \\
\hline 5 & Inclusión & 2 & Inclusión \\
\hline 6 & Justicia social & 3 & Inclusión \\
\hline 7 & Otras minorías & 1 & Inclusión \\
\hline 8 & Exposiciones dirigida a & 1 & Inclusión \\
\hline 9 & población local inmigrante & 54 & Inclusión \\
\hline
\end{tabular}

Tabla 9. Resultados de temas y contenidos para el grupo temático de las TIC. 


\begin{tabular}{|c|c|c|c|}
\hline $\begin{array}{l}\text { No temas y } \\
\text { contenidos }\end{array}$ & Temas y contenidos & $\begin{array}{c}\text { № } \\
\text { artículos }\end{array}$ & Grupo temático \\
\hline 1 & TIC & 14 & TIC \\
\hline 2 & Teoría de la transformación digital de los museos & 15 & $\mathrm{TIC}$ \\
\hline 3 & App móviles & 3 & $\mathrm{TIC}$ \\
\hline 4 & Exposición virtual & 2 & $\mathrm{TIC}$ \\
\hline 5 & $3 \mathrm{D}$ & 2 & $\mathrm{TIC}$ \\
\hline 6 & Realidad aumentada & 2 & $\mathrm{TIC}$ \\
\hline 7 & Redes sociales & 2 & $\mathrm{TIC}$ \\
\hline 8 & Instalación lumínica digital & 1 & $\mathrm{TIC}$ \\
\hline 9 & TIC inmersiva & 1 & $\mathrm{TIC}$ \\
\hline 10 & Emprendimiento & 1 & $\mathrm{TIC}$ \\
\hline 11 & Compromiso o fidelización con el museo & 1 & $\mathrm{TIC}$ \\
\hline 12 & Estudios de público & 1 & $\mathrm{TIC}$ \\
\hline 13 & Mediación digital & 1 & $\mathrm{TIC}$ \\
\hline 14 & Musicalización digital & 1 & $\mathrm{TIC}$ \\
\hline 15 & Códigos QR & 1 & $\mathrm{TIC}$ \\
\hline 16 & Realidad virtual & 1 & $\mathrm{TIC}$ \\
\hline 17 & Robots & 1 & $\mathrm{TIC}$ \\
\hline 18 & Visita Remota & 1 & $\mathrm{TIC}$ \\
\hline & Subtotal de artículos & 51 & $T I C$ \\
\hline
\end{tabular}

Tabla 10. Resultados de temas y contenidos para el grupo temático de Participación.

\begin{tabular}{|c|l|c|c|}
\hline \multicolumn{1}{|c}{$\begin{array}{l}\text { № temas y } \\
\text { contenidos }\end{array}$} & \multicolumn{2}{c}{$\begin{array}{c}\text { № } \\
\text { artículos }\end{array}$} & Grupo temático \\
\hline 1 & Co-comisariado & 10 & Participación \\
\hline 2 & Participación digital & 7 & Participación \\
\hline 3 & Interacción & 5 & Participación \\
\hline 4 & Teoría de la participación & 5 & Participación \\
\hline 5 & Jóvenes & 3 & Participación \\
\hline 6 & $\begin{array}{l}\text { Museos en colaboración con las comunidades } \\
\text { sociales }\end{array}$ & 3 & Participación \\
\hline 7 & Colaboración inter-institucional e inter-profesional & 2 & Participación \\
\hline 8 & Familias & 2 & Participación \\
\hline 9 & Participación a través del libro de visitas & 2 & Participación \\
\hline 10 & Los visitantes producen contenido en la exposición & 2 & Participación \\
\hline 11 & $\begin{array}{l}\text { Aplicación educativa de las colecciones digitales de } \\
\text { museos }\end{array}$ & 1 & Participación \\
\hline 12 & Juegos & 1 & Participación \\
\hline & & 43 & Participación \\
\hline
\end{tabular}

Tabla 11. Resultados de temas y contenidos para el grupo temático de Otros.

\begin{tabular}{|c|c|c|c|}
\hline $\begin{array}{l}\text { № temas y } \\
\text { contenidos }\end{array}$ & Temas y contenidos & $\begin{array}{c}\text { № } \\
\text { artículos }\end{array}$ & Grupo temático \\
\hline 1 & Política de museos & 15 & Otros \\
\hline 2 & Eco-museos & 8 & Otros \\
\hline 3 & Pandemia por COVID-19 & 4 & Otros \\
\hline & Subtotal de artículos & 27 & Otros \\
\hline
\end{tabular}

